\section{Unsolicited/solicited marketing}

Why this matters
What happened

\section{Privacy tools challenge profiling}

Why this matters
The Guidance contains some very logical and legalistic statements as to what is solicited and unsolicited marketing. Again, this is an area where more examples would help to clarify the ICO's view. The DMA accepts that organizations need to have clear wording on their data collection statements, and therefore consumers know what they are signing up to.

So far, the ICO has responded positively to the DMA's comments. It is important that the Direct Marketing guidance is as clear as it can be so that organizations can understand the ICO's position.

James Milligan, legal and public affairs adviser, The Direct Marketing Association (UK)

\section{Will cookie alternatives sidestep privacy laws?}

\section{Mark Webber}

Journal of Direct, Data and Digital Marketing Practice (2014) 15, 245-247. doi:10.1057/dddmp.2013.74

Who: The UK's ICO

Where: London, UK

When: 6 November 2013

Law stated as at: December 2013

Over the past year, there has been increasing press and social media speculation that the 'tech giants' are developing technological alternatives to web browser cookies.

This is intended partly to improve user tracking across platforms browser-based cookies do not help a marketer trying to follow behaviour across smart TV, games console, smartphone, app and tablet interactions where cookies are not king. However, equally, it is partly a direct consequence of the sometimes negative attention paid to cookie-based behavioural advertising and user-tracking techniques.

Many in the technological and advertising industries understand that increased privacy education and awareness, together with more opt-out and privacy-monitoring tools, will increasingly interfere with the art of making behavioural inferences and building profiles based solely on the humble cookie. What is more, the future EU Data Protection Regulation looks likely to regulate the building of personal profiles potentially even if the data does not directly relate to an individual. Computer Weekly went as far as declaring cookie tracking an 'outdated approach' last month.

Of course, replacing the browser cookie with a proprietary tracking technology may also ring-fence advertising and behavioural tracking 


\section{PECR still rules}

\section{Do privacy policies go far enough?}

\section{New technology solutions}

within a certain technology vendor's ecosystem. If Microsoft's own technology can track user behaviour within the Microsoft environment to the exclusion of others, it does not take much to realize that some genuine market advantage is available. However, if that proprietary tracking technology could also bypass existing privacy laws, particularly those around cookies, could the world's technological and advertising giants perhaps steal a march on current stringent EU laws?

No so! On 6 November 2013, an ICO spokesperson confirmed that any technology developed to track individuals online would be required to comply with 'all relevant aspects' of UK law. Therefore, we turn back to Data Protection Act and the Privacy and Electronic Communications Regulations (PECR) and principles of transparency and control.

The news in 2003 and then again in 2011 and 2012 saw endless coverage around 'the new cookie law'. Driven by Europe, the UK's Privacy and Electronic Communications (EU Directive) Amendment Regulations 2011 (the 'Regulations') implemented the required specific changes in the UK law. No longer was it sufficient to inform and offer information on how to opt-out of a cookie. The 2011 revisions meant that cookies or similar devices must not be used unless the subscriber or user of the relevant terminal equipment:

(a) is provided with clear and comprehensive information about the purposes of the storage of, or access to, that information; and

(b) has given his or her consent.

The law already referred to 'similar devices' and simply regulates the 'storage of' or 'access to' information. In fact, in the full text of Section 6 of the Regulations, the word 'cookie' does not even appear. Thus, the so-called 'cookie legislation' has a wider reach. This 'cookie law' is already technologically neutral.

But is any of this news? We already have web beacons, clear pixel gifs and HTML5 local storage, not to mention Adobe's proprietary 'Flash Cookie' (another form of local object storage), all of which are already deployed (sometimes in conjunction with a browser cookie) to store and track user's data and information. Just as lawyers focus on IP addresses as identifiers, but neglect to question about numerous others, we see a similar trap within tracking compliance and privacy policy awareness. 'Browser cookies' get a mention, perhaps a generic nod to a web beacon, but how many privacy policy disclosures go further?

Newer technologies, such as javascript tagging or coding to assemble device IDs and 'device fingerprinting', are not as apparent as our humble cookie. With new technology and tracking also clearly at development stage, now is the time to dispel the rumour that future tracking will fall outside the current law. However, it is also the time to ask more questions. 
And, quelle surprise, the ICO has already brought this to our attention, way back when it published its May 2012 Cookie Guidance, which reminded us:

In some areas, it is possible for functions usually performed by a cookie to be achieved through other means. This could include, for example, using certain characteristics to identify devices so that you can analyse visits to a website (this is sometimes known as 'device fingerprinting') ... Focusing solely on cookies is missing the point. Even where the clear cookies rules do not apply, you must consider the DPA whenever you are collecting information that builds up a picture that could allow you to identify an individual. You should tell people what you are collecting and how you are using this information.

(http://www.ico.org.uk/for_organisations/privacy_and_electronic_ communications/the_guide/cookies)

Another timely reminder that it is not about cookies, it is about privacy.

Mark Webber, partner, Osborne Clarke

\title{
German federal court rules against 'tell-a-friend'
}

\author{
Ulrich Baumgartner and Johannes Baumann
}

Journal of Direct, Data and Digital Marketing Practice (2014) 15, 247-248. doi:10.1057/dddmp.2013.75

Who: German Federal Court of Justice

When: 12 September 2013

Where: Germany

Law stated as at: November 2013

What happened

Unfair competition laws apply
According to a ruling of the German Federal Court of Justice ('Bundesgerichtshof'), dated 12 September 2013, German companies are no longer allowed to use 'tell-a-friend' functionalities on their websites. The court considers emails sent via such functionalities as unsolicited marketing messages (ie, spam emails) for which the company providing such tell-a-friend functionality on its website is responsible. In the absence of an expressing and prior consent of the recipient of such a message (which will usually not exist in practice), the use of such functionalities might therefore lead to cease-and-desist requests and fines.

Under German laws against unfair competition, and subject only to few exceptions, any form of email marketing without expressing prior consent of the addressee is unlawful and can be legally challenged by, for example, 